\title{
Short-term memory for figural items as a function of the number of variable dimensions
}

\author{
STEFAN SLAK \\ University of Toledo, Toledo, Ohio
}

\begin{abstract}
A total of 108 undergraduates were given a memory span task for figural items varying in one, two, or three dimensions. Memory span in item units significantly decreased, and memory span in bits of information significantly increased as functions of the increase in the number of variable dimensions and bits of information per item. Results are interpreted as additional evidence that the constant chunk hypothesis is wrong and as further extension of previous data on univariate and bivariate information storage.
\end{abstract}

In summarizing some of the data on unidimensional and multidimensional absolute judgments, Miller (1956) noted that the channel capacity depends not only on a particular dimension used but also on the number of simultaneously used dimensions. The channel capacity of human observers increases as the number of simultaneously variable dimensions increases. McCormick (1970, p. 91) also states that, in combinations of dimensions, the maximum information that can be transmitted is noticeably greater, ranging from 3.5 bits to as high as 7.2 bits. Beebe-Center, Rogers, and O'Connell (1955), for example, measured the channel capacity of human observers for concentrations of sucrose alone, salt alone, and sucrose and salt combined and found that the channel capacity in bits of information was 1.7 for salt alone and sucrose alone, and 2.4 for the two combined. Slak's (1969) review of the available data suggested an increase in the channel capacity with additional dimensions, but at a decreasing rate. It also has been noted that information transmission rate in memory processes is inferior to that in perception (Weltner, 1981).

This effect of dimensionality led Slak (1971) to test memory span for univariate and bivariate figural items (with color and shape as dimensions). He found that the memory span in bits of information was higher in the bivariate condition, but the span measured in terms of items was lower in the bivariate condition as compared with the univariate condition. Replication with different dimensions yielded the same results (Slak, 1974). Because bivariate items had twice as high information content in bits as univariate items had, the results showed that memory span was limited neither to a given amount of information nor to a given number of items or chunks. Although Miller (1956) prematurely concluded that memory span depends on number of items or chunks (constant chunk hypothesis) regardless of information content, Slak's data showed that memory span is conjointly

The author's mailing address is: Department of Psychology, The University of Toledo, 2801 W. Bancroft St., Toledo, OH 43606. limited by the number of chunks and by the amount of information contained in those chunks.

A different experimental paradigm resulted in the same conclusion. Slak, Hirsch, and Syrja (1979) instructed the subjects to randomize univariate or bivariate items. Redundancy (inverse of the proportion of maximum possible information) was higher in the bivariate condition, showing that items containing more information are more difficult to randomize, but the information production rate (total information produced per unit of time) was higher in the bivariate condition. Again, the information processing ability increased with increased information per item, but at a slower rate than the increase in information per item (as the redundancy data indicate).

The third experimental paradigm yielding analogous results was phonemic recoding of figural items (Slak, 1972; Slak, Kelley, \& Skibski, 1979). In the control condition, subjects learned to name the two dimensions used with two natural language words, and in the experimental condition they learned to name them with a twophoneme syllable: a consonant, standing for one dimension, and a vowel, standing for the other dimension. The prelearned code reduced the length of verbal representation of the same amount of information. Such reduction in verbal representational redundancy led to an increase in memory span for figural items, but the increase in memory span was inferior to the amount of decrease in representational redundancy. The preceding results are related to similar earlier experiments with phonemic recoding of digital information (Slak, 1970) and to earlier work of Glanzer and Clark (1962) who noticed that information representable with fewer words is more easily processed.

In the above examples, two types of chunking are involved. In the studies on univariate and bivariate items (e.g., Slak, 1971), items may simultaneously vary in two dimensions. An example of unidimensional variation is: "red," " blue," "'green," ... or "triangle," "square," "star," ... An example of bivariate sequence is: "red triangle," "green star," "blue square," ... (actual figures and colors, not words). Such combining of two dimensions into a single item may be called stimulus 
chunking, because the chunking is done before items are presented to the subject. A chunk in this case is a new gestalt, presumably perceived as a unit rather than as a set of components. In the studies on phonemic recoding, on the other hand, the subject learns the phonemic code first so that he/she can recode the items into a much shorter verbal representation than the one provided by the natural language. For two dimensions, line orientation and the number of lines, the natural language representation of "two horizontal lines" can be encoded phonemically as "may" (Slak, 1979). In this case, the chunking is done by the subject according to a prelearned code and has been called the process of reduction of representational redundancy.

The purpose of the present study was to extend the findings on the effect of stimulus chunking from two dimensions to three dimensions. It was predicted that as the number of simultaneously varying dimensions increases from one to two to three, the memory span in bits of information increases and the memory span in item units decreases as a function of number of dimensions.

\section{METHOD}

\section{Subjects}

A total of 108 volunteer undergraduate students of both sexes were randomly assigned to three experimental conditions of 36 subjects each.

\footnotetext{
Apparatus

A Hunter timer for control of stimulus presentation and intertrial interval was connected to a slide projector for presentation of stimuli on a screen about $3 \mathrm{~m}$ distant from the subject.

Design

The design was a unifactorial one with independent groups for the three experimental conditions: univariate, bivariate, and trivariate.

\section{Procedure and Tasks}

In a given trial, a subject was presented with a sequence of figural items on a screen. Each item was presented for $2 \mathrm{sec}$ with 1-sec intertrial intervals. The sequence length increased from 2 to 12 items, each length presented once. After each sequence presentation, the subject recalled at his/her own pace all the items he/she could by drawing them in a column with the same number of cells as the sequence length.

In the univariate condition, the items varied in one of the three dimensions: orientation of line (horizontal or vertical), number of lines (one or two parallel), and shape of lines (straight or S-curved). Each of the three possible variable dimensions was used with 12 of the 36 subjects assigned to the condition. Each of the 12 subjects for a given variable dimension was given a different combination of the variable dimension and the values of other fixed dimensions. The order of items in a list was completely randomized.

In the bivariate condition, items varied along two dimensions (orientation plus number; orientation plus shape; or number plus shape). There were six possible combinations of two variable dimensions and values of the fixed dimension, each one used with 6 of the 36 subjects in the condition. The order of items in a list was completely randomized.

In the trivariate condition, all three dimensions were variable (one combination) yielding eight possible stimulus alternatives completely randomized for all 36 subjects in the condition.

In the univariate condition, each item was worth one bit of information (two alternatives); in the bivariate condition, two bits of information (four alternatives); and in the trivariate condition, three bits of information (eight alternatives).
}

\section{Scoring}

For each subject, memory span for sequence length was determined by adding the number of sequence lengths correctly reproduced to a value of 1.5 ( 2 was the shortest sequence length used). This addition yielded memory span in item units. To obtain memory span in bits of information, the memory span in item units was multiplied by 1 in the univariate condition, by 2 in the bivariate condition, and by 3 in the trivariate condition.

\section{RESULTS AND DISCUSSION}

The average memory span in terms of items for univariate, bivariate, and trivariate conditions was $8.11,5.08$, and 4.02, respectively. The overall differences between conditions were significant $[\mathrm{F}(2,105)=27.69, \mathrm{p}<.01]$. Differences between each two conditions were also significant [univariate vs. bivariate, $\mathrm{F}(1,105)=11.4$, $\mathrm{p}<.01$; univariate vs. trivariate, $\mathrm{F}(1,105)=172.49$, $\mathrm{p}<.01 ;$ and bivariate vs. trivariate, $\mathrm{F}(1,105)=11.53$, $\mathrm{p}<.01]$.

In terms of bits of information, the average memory span for uni-, bi-, and trivariate conditions was 8.11, 10.16 , and 12.06 , respectively. The overall differences were significant $[\mathrm{F}(2,105)=27.69, \mathrm{p}<.01]$. The differences between individual means were also significant $[\mathrm{F}(1,105)=14.83,55.40$, and 12.90 , respectively, $(\mathrm{p}<.01)]$.

In conclusion, memory span in item units significantly decreased and memory span in bits of information significantly increased as a function of the increase in the number of variable dimensions from one to three. The results are consistent with previous data on univariate and bivariate dimensions and represent extension of findings to three dimensions.

The data of this experiment show again that the popular constant chunk hypothesis based on indirectly reported data obtained from a single subject (Miller, 1956) and already part of the "introductory psychology" culture (e.g., Rumelhardt, 1977, p. 177) is wrong. Memory span does not depend merely on the number of items or chunks, but also on the amount of information contained in them.

\section{REFERENCES}

Beebe-Center, J. C., Rogers, M. S., \& O'Connell, D. N. (1955). Transmisson of information about sucrose and saline solutions through the sense of taste. Journal of Psychology, 39, 157-160.

GlanZer, M., \& ClaRK, W. H. (1962). Accuracy of perceptual recall: An analysis of organization. Journal of Verbal Learning \& Verbal Behavior, 1, 289-299.

McCoRmick, E. J. (1970). Human factors engineering (3rd ed.). New York: McGraw-Hill.

MilleR, G. E. (1956). The magical number seven plus or minus two: Some limits on our capacity for processing information. Psychological Review, 63, 81-97.

RUMELHARDT, D. E. (1977). Introduction to human information processing. New York: Wiley.

SLAK, S. (1969). Multidimensional information transmission as a function of unidimensional channel capacity. Psychological Review, 76, 224-226.

SLAK, S. (1970). Phonemic recoding of digital information. Journal of Experimental Psychology, 86, 398-406. 
SLAK, S. (1971). Univariate and bivariate information storage. American Journal of Psychology, 84, 205-209.

SLAK, S. (1972). Efecto de la recodificación fonémica sobre la amplitud de la memoria inmediata. Revista Latinoamericana de Psicología, $\mathbf{4}$, 23-33.

SLAK, S. (1974). Almacenamiento univariado y bivariado de información para dígitos y localizaciones. Revista Latinoamericana de Psicología, 6, 341-346.

Slak, S., Hirsch, K. A., \& SyrJa, K. V. (1979). Randomization of univariate and bivariate sequences and Baddeley's limited capacity hypothesis. Journal of Psychology, 103, 169-182.
Slak, S., Kelley, K. M., \& Skibski, J. (1979). Phonemic recoding of figural information and memory span. Bulletin of the Psychonomic Society, 14, 304-306.

WELTNER, K. (1981). La mezurado de la subjektiva informo en instruajo kay la konstato de la informredukto ce problemsolvaj procezoj. In Kibernetiko de la homa lingvo kaj aliaj internacilingvaj aktoj de la IX. - Internacia Kongreso de Kibernetiko (pp. 19-29). BudapestPadeborn: Hungara Esperanto-Asocio, Institutio pri Kibernetiko.

(Manuscript received for publication April 23, 1985.) 